\title{
Retos de los ordenamientos jurídicos nacionales hacia la implementación del Enfoque por Ecosistemas
}

\author{
Challenges of national legal systems towards \\ the implementation of the Ecosystem Approach \\ Os desafíos do sistema jurídico nacional até \\ a implementação da aproximação do ecossistema
}

\begin{abstract}
RESUMO
No artigo propomos uma revisão sobre os desafios que impõem o enfoque de controle pelos ecossistemas para organizações jurídicas nacionais, fazendo-se notar que este corresponde com uma revisão integral e sistemática do desenvolvimento. Portanto, existem varios modelos que obedecem a este enfoque, entre os cuais se encontram, o controle integrado de aguas e áreas costeiras. Isto pode facilitar a abordagem dos problemas que afetam aos territorios marinho costeiros tendo em vista os impactos negativos, cuja as causas devem ser controladas desde as gerências altas das bacias hidrográficas, ou seja com uma visão mais complexa e integrada dos problemas das suas causas e consequêcias.
\end{abstract}

\section{PALAVRAS-CHAVES}

Ecossistemas marinho-costeiros, Enfoque pelos ecossistemas, Produtos e serviços ambientais, Sustentabilidade, Política de desenvolvimento, Princípios do EE.

\section{RESUMEN}

En el artículo se hace una revisión sobre los retos que imponen el enfoque de manejo por ecosistemas para los ordenamientos jurídicos nacionales, haciendo notar que este corresponde con una visión integral y sistemática del desarrollo, así mismo existen varios modelos que obedecen a este enfoque, entre los cuales se encuentra, el manejo integrado de aguas y áreas costeras y el manejo integrado de zonas costeras.

Esto puede facilitar el abordaje de las problemáticas que afectan a los territorios marino-costeros teniendo en cuenta los impactos negativos, cuyas causas deben ser manejadas desde las partes altas de las cuencas hidrográficas, es decir, con una visión más compleja e integradora de los problemas, sus causas y consecuencias.

\section{PALABRAS CLAVE}

Ecosistemas marino-costeros, Enfoque por Ecosistemas, Bienes y servicios ambientales, Sostenibilidad, Políticas de desarrollo, Principios del EE.

\begin{abstract}
This article reviews the challenges posed by the ecosystem management approach issued by the national law, noting that this corresponds to a comprehensive and systematic approach to development; also there are several models which follow this approach, including which is, integrated water management and integrated coastal areas and coastal zone management. This can facilitate addressing the problems affecting marine and coastal areas taking into account the negative impacts; the causes must be managed from the upper reaches of the watershed, that is, with a more complex and integrated view of problems, its causes and consequences.
\end{abstract}

\section{KEYWORDS}

Coastal marine ecosystems Ecosystem Approach, EGS, Sustainability, Development policies, Principles of EE.

YAMILKA CARABALLO DÍAZ

Centro de Desarrollo Local y Comunitario. Ministerio de Ciencia, Tecnología y Medio Ambiente. Cuba. Universidad de Bue-

nos Aires.yamicadi@cedel.cu

CÉSAR A. IPENZA PERALTA

Especialista en temas ambientales del Perú. Centro Peruano de Estudios Sociales-CEPES. cipenza@yahoo.es 


\section{INTRODUCCIÓN}

La gobernanza de espacios marino-costeros es especialmente compleja dada las características particulares de estos territorios. En tal sentido uno de los aspectos que determinan esta complejidad es el hecho de que la zona costera constituye un espacio de interfase entre la atmósfera, la tierra y el mar (Cincin-Saint y Knecht, 1998). Por tanto, en ella confluyen procesos naturales propios de intercambios de energía y materia, cuyas causas y dinámicas provienen tanto de los espacios marinos como de los terrestres.

Ejemplo de ello en el caso de las zonas tropicales, es que los procesos de filtración de las aguas terrestres proporcionan la calidad para el desarrollo de los arrecifes coralinos, los que forman ecosistemas de muy alta productividad y servicios que garantizan, junto con los bosques costeros y los pastos marinos, el reclutamiento de especies marinas y costeras, que constituyen la tasa de reemplazo de las poblaciones marinas. Por otra parte, los bosques costeros evitan la penetración de la cuña de intrusión salina, proporcionando una estabilidad en toda la zona costera que posibilita la alta diversidad de paisajes (MEA, 2005).

Dada esta interacción entre la tierra y el mar es que se plantea que "El funcionamiento de una cuenca o un área marino-costera, como todo sistema complejo, debe ser comprendido atendiendo de forma integral a todas sus partes, componentes y procesos, tanto naturales como construidos" (Brito, 2010) Un dato interesante en este sentido es que en América Latina el $86 \%$ de las aguas residuales llega sin tratar a ríos y océanos y en el Caribe la cifra aumenta al 80-90 \% (PNUMA, 2010). Siendo así, la contaminación es una de las amenazas de los ecosistemas marinocosteros cuyo origen, en muchos casos, tiene lugar en la parte alta de las cuencas hidrográficas, lo que indica que una gestión efectiva de esta problemática en la zona costera debe necesariamente tener en cuenta el entramado de usuarios e instituciones de la cuenca tributaria en cuestión.

Además, estas zonas funcionan de un modo cualitativamente diferente al funcionamiento natural, como consecuencia del impacto que la sociedad le ocasiona en su interacción práctica, es decir, por la forma en que se asimilan por los ecosistemas costeros las transformaciones humanas y a su vez se revierten los resultados de esto sobre el hombre (Miranda, 2003). Por eso se plantea que los problemas constituyen el origen de la mayor parte de los conflictos en la zona costera y suelen ser de dos tipos, los que afectan a los recursos y los específicos relacionados con su gestión y administración (Barragán, 2005).

De ahí que se considere que

la participación local en la gestión de los recursos naturales se asocia con el establecimiento de sistemas de gobernanza y arreglos institucionales que favorecen el uso sostenido de los recursos, al mismo 
tiempo que aseguran los medios de vida de poblaciones en zonas rurales (Pacheco et al., 2008).

Se considera la gobernanza como "los mecanismos relacionales mediante los cuales los actores articulan sus intereses, ejercen sus derechos y obligaciones y median en los desacuerdos y conflictos" (Salvador, 2010).

Por otra parte, el manejo tradicional de los recursos marinos y costeros se ha caracterizado por un enfoque sectorial, por ejemplo, los recursos pesqueros se han manejado de forma separada al desarrollo de la explotación de petróleo y gas (Cincin-Saint \& Knecht, 1998), aunque ambos recursos coexistan en un territorio determinado. Además, se plantea que "lo más complejo, para lograr la compatibilización y coordinación de intervenciones en las cuencas y zonas marino-costeras, es que hay cientos de decisiones y acciones, que proceden de actores e instituciones que juegan roles muy diferentes (Brito, 2010). Por esta razón, representa un gran desafío para la gobernanza de las zonas marino-costeras la adopción e implementación de los principios del Enfoque de Manejo por Ecosistemas, como es el caso del enfoque de Manejo Integrado de Aguas y Áreas Costeras, de modo que se propicie una visión integradora en la gestión de recursos hídricos y costas, así como de otros recursos naturales y el desarrollo económico social en sentido general (PNUMA, 2010).

Lo anterior se corresponde con el plantea- miento de que el manejo de la gestión pública de este tipo de espacios debe reorientarse con un enfoque más complejo pues

el territorio es mucho más que mero soporte físico o contenedor de actividades. Es el espacio donde se producen relaciones sociales y económicas. Por tanto, debe ser entendido como recursos, como patrimonio, como paisaje cultural, como expresión de la memoria colectiva, como bien público, como espacio de solidaridad y como legado.

Por eso resulta de particular importancia revisar los modelos tradicionales de gobierno, pues "ya no se trata sólo de reducir la distancia, sino de cambiar la forma de relación entre gobierno y ciudadano". Así es que se considera que
de los enfoques tradicionales centrados en la ordenación del territorio y en la preemi- nencia de las políticas sectoriales, se debe transitar ahora hacia visiones más integra- das o sistémicas. Las decisiones se deben tomar de forma más participada y las po- líticas deben ser más respetuosas con los contextos específicos y con la identidad, la cultura y la memoria colectiva de los dife- rentes lugares (Castillo et al., 2010).

De modo que un modelo de gobernanza de la zona costera debe concebirse no solo su complejidad como espacios físico-geográficos y características específicas de los procesos naturales que en estos ocurre, sino tam- 
bién, y de forma muy particular, teniendo en cuenta las necesidades y realidades de los procesos económicos y sociales que en estos se desarrollan. En tal sentido, resulta de particular importancia analizar los retos de los ordenamientos jurídicos nacionales hacia la implementación del Enfoque por Ecosistemas, en tanto principios que pueden posibilitar la gestión de los ecosistemas costeros considerando las particularidades de los procesos naturales, económicos y sociales que en estos se desarrollan. Es por ello que el presente artículo se centra en comentar dichos retos, específicamente en lo relacionado con la participación de las comunidades indígenas y locales en la adopción de decisiones relacionadas con el uso de los recursos naturales, la institucionalidad ambiental y la valoración económica de bienes y servicios ambientales.

1. DE LA TUTELA JURÍDICA DEL MEDIO AMBIENTE HACIA LA TUTELA JURÍDICA DE LA SOSTENIBILIDAD DEL DESARROLLO

Se considera que la evolución del Derecho ambiental ha estado marcada por tres hitos fundamentales que cronológicamente se manifiestan en la Declaración de Estocolmo sobre Medio Ambiente Humano*, la Decla-

* En su Principio 4 establece que: "Al planificar el desarrollo económico debe atribuirse importancia a la conservación de la naturaleza, incluidas la flora y la fauna silvestres" y el Principio 13 establece que: "A fin de lograr una más racional ordenación de recursos y mejorar así las condiciones ambientales, los estados deberían adoptar un enfoque integrado y coordinado de la planificación del desarrollo, de modo que quede asegurada la compatibilidad del desarrollo con la necesidad de proteger y mejorar el medio humano en beneficio de su población". ración de Río sobre Medio Ambiente ${ }^{* *}$ y Desarrollo, y la Declaración de Johannesburgo sobre Desarrollo Sostenible*. En los tres, de una u otra forma se hace notar la necesidad de equilibrio en la relación hombre-naturaleza en el diseño de las políticas de desarrollo teniendo en cuenta la interdependencia que existe entre la sociedad y el escenario geográfico-espacial en que esta se sustenta.

Un ejemplo de la evolución del concepto en Acuerdos Ambientales Multilaterales lo constituye la extensión del alcance de aplicación de la Convención Relativa a los Humedales de Importancia Internacional Especialmente como Hábitat de Aves Acuáticas, conocida como Convención Ramsar, cuyo objetivo inicial estaba dirigido a la conservación y uso racional de los humedales como hábitat de las especies acuáticas, pero que en la actualidad reconoce la importancia de este ecosistema en la conservación global y el ciclo hidrológico, por sus funciones ecológicas, los valores que representan para el patrimonio cultural y usos tradicionales, así como para la diversidad biológica en general (García y Rey, 2005).

** En tal sentido es importante hacer notar que el Principio 4 de la Declaración de Río sobre Medio Ambiente y Desarrollo establece que: "A fin de alcanzar el desarrollo sostenible, la protección del medio ambiente deberá constituir parte integrante de la protección del proceso de desarrollo y no podrá considerarse en forma aislada" y en su Principio 7 establece que: "Los Estados deberán cooperar con espíritu de solidaridad mundial para conservar, proteger y restablecer la salud y la integridad del ecosistema de la Tierra".

* Se pronuncia por "la responsabilidad colectiva de promover y fortalecer, en los planos local, nacional, regional y mundial, el desarrollo económico, desarrollo social y la protección ambiental, pilares interdependientes y sinérgicos del desarrollo sostenible". 
También el Convenio sobre la Diversidad Biológica (CBD) adoptado en Nairobi el año de 1992, el cual es uno de los dos instrumentos ambientales internacionales, legalmente vinculantes, abiertos para la firma de la comunidad internacional en la Cumbre de Río, el 5 de junio de 1992 (Ipenza, 2010), constituye un elemento clave de este tránsito hacia el Desarrollo Sostenible. En tal sentido, la Decisión Sexta de la Quinta Conferencia de las Partes, efectuada en Nairobi, Kenya, en mayo del año 2000, aprobó el Enfoque por Ecosistemas $(\mathrm{EE})^{*}$ concibiéndolo a este como "una Estrategia para el manejo de la tierra, el agua y los recursos vivos, que promueve la conservación y uso sostenible en forma equitativa, basada en la aplicación de los métodos científicos pertinentes" (CBD, 2000).

El EE introduce cambios en la concepción sobre el ordenamiento y gestión de los recursos naturales en el sentido de evolucionar de espacios individuales a ecosistemas, de pequeñas a mayores escalas espaciales, de

* El Convenio de Diversidad Biológica ha abordado el Enfoque por Ecosistemas en varias Decisiones. Por ejemplo, en la Decisión Primera de la Cuarta Conferencia de las Partes "Informe y recomendaciones de la tercera reunión del Órgano Subsidiario de Asesoramiento Científico, Técnico y Tecnológico (OSACTT), e instrucciones de la Conferencia de las Partes al Órgano Subsidiario de Asesoramiento Científico, Técnico y Tecnológico", en el apartado B) se precisa que la Conferencia de las partes solicita al OSACTT elabore principios y otras orientaciones acerca del enfoque por ecosistemas como resultado de lo cual se adoptan los Principios del Enfoque por Ecosistemas mediante la Decisión Sexta de la Quinta Conferencia de las Partes. Posteriormente, se da seguimiento a la incorporación de estos principios en las estrategias y legislación nacional, mediante la Decisión Decimosegunda de la Sexta Conferencia de las Partes, la Decisión Decimoprimera de la Séptima Conferencia de las Partes y la Decisión Séptima de la Novena Conferencia de las Partes. mediano a largo plazo, de hombre independiente del ecosistema a hombre como parte del ecosistema, de manejo divorciado de la ciencia a manejo adaptativo, de manejo por uso de recurso a manejo en función de la capacidad del ecosistema para producir bienes y servicios ambientales (Lubchenko, 1994; Sherman and Duda, 1999).

EI EE busca un balance entre la conservación de la diversidad biológica, su uso sostenible y las necesidades asociadas al desarrollo humano cuyas alternativas dependen de los bienes y servicios ambientales que brindan un determinado sistema natural. Es una estrategia para la gestión integrada de los recursos de tierras, hídricos y vivos que promueve la conservación y la utilización sostenible en forma equitativa. Por ello se ha reconocido que uno de los mayores retos de la comunidad internacional, los Estados y las comunidades locales es "mantener los servicios ecosistémicos necesarios y conciliar el uso de la tierra y los recursos hídricos con otros usos" (PNUMA, 2007).

En correspondencia con ello se han promovido diversos modelos de gestión de recursos naturales que obedecen a un EE, tales como: Manejo Integrado de Recursos Hídricos, Manejo Integrado Costero, Manejo Integrado de Cuencas Hidrográficas, Manejo Integrado de Aguas y Áreas Costeras y Manejo Sostenible de Tierra. También se considera que la gestión de Áreas Naturales Protegidas constituye un ejemplo de $\mathrm{EE}$, en ese sentido el $\mathrm{EE}$ contribuye de manera significativa al logro 
de los objetivos del Plan deTrabajo de Áreas Protegidas del CBD y a reducir la pérdida de biodiversidad, especialmente para los corredores de conservación o corredores biológicos.

Los 12 principios que lo conforman son complementarios y están relacionados entre sí. Es el sistema de principios completo, más que cada uno de los principios visto de manera aislada, lo que conforma y le da valor agregado al EE, aunque no siempre resulta posible constatar la aplicación de todos estos en una realidad determinada.

Se plantea que estos principios incluyen aspectos ecológicos, sociales y económicos y el enfoque pone énfasis en gobernanza y equidad social. Las ventajas de contar con un marco común, como el que plantea el EE, tienen que ver con la posibilidad de articular los múltiples y dispersos esquemas de gestión integrada de los recursos naturales; coordinar y promover sinergias entre los diferentes convenios, estrategias y planes de acción sectoriales; fortalecer el desarrollo y validación en la práctica de métodos de manejo integrado; así como dotar a los gestores de una herramienta integral y adaptable al contexto para la implementación a diferentes escalas de las políticas y estrategias nacionales sobre recursos naturales (Guerrero, 2004). Estos deben ser considerados desde las primeras etapas de elaboración de políticas y de planificación en todos los niveles que tienen relación con los recursos naturales o que in- ciden en ellos y que pueden ser útiles en las estrategias de reducción de la pobreza y en políticas y planificación a diferentes niveles y especialmente de forma intersectorial (CBD OSACTT, 2007).

EI EE implica el entendimiento de procesos ecológicos y socioculturales de modo que mediante su aplicación se puede promover el desarrollo sostenible, en tanto ofrece una visión integral orientada hacia el suministro continuo de bienes y servicios ambientales mediante el mantenimiento de procesos ecológicos esenciales y la participación activa de los sectores involucrados en su gestión (Waltner-Toews and Kay, 2005).

Se plantea que "los ecosistemas aportan servicios ambientales que además de críticos son valiosos para las poblaciones, en lo local, nacional, regional y global; y se ubican entre los mayores contribuyentes al bienestar humano" (PNUMA, 2010). En tal sentido, el Convenio sobre la Diversidad Biológica considera que los servicios que brindan los ecosistemas se clasifican en cuatro grupos:

- Servicios de aprovisionamiento, o suministro de bienes que benefician directamente a las personas y suelen tener un claro valor monetario, como la leña de los bosques, las plantas medicinales y los peces de los mares, ríos y lagos;

- Servicios reguladores, son la gama de funciones vitales desempeñadas por los ecosistemas a las que en raras ocasiones se les asigna un valor monetario en los 
mercados convencionales. Entre ellos se cuentan la regulación del clima mediante el almacenamiento de carbono y el control de las precipitaciones locales, la eliminación de contaminantes por medio del filtrado del aire y las aguas, y la protección frente a los desastres, como el deslizamiento de tierras y las tormentas costeras;

- Servicios culturales, que no ofrecen beneficios materiales directos pero contribuyen a satisfacer ciertas necesidades y deseos más amplios de la sociedad y, por lo tanto, inciden en la predisposición de las personas a costear los gastos de la conservación. Entre otros, cabe mencionar el valor espiritual que se da a ciertos ecosistemas, como las arboledas sagradas, y la belleza estética de los paisajes o las formaciones costeras que atraen a los turistas; $y$

- Servicios de apoyo, que no benefician directamente a las personas pero son esenciales para el funcionamiento de los ecosistemas y, por ende, responsables indirectos de los demás servicios. Entre ellos se cuentan la formación de suelos y los procesos de crecimiento de las plantas.

No se trata de un enfoque de "no tocar" los recursos naturales, sino más bien de ordenar su uso previendo que el cambio de los ecosistemas debido a las actividades humanas es inevitable pero que dicho cambio no debe rebasar su límite de resiliencia, porque a la vez genera "consecuencias directas, indirectas, positivas y negativas, sobre el bienestar humano en aspectos, como por ejemplo el acceso a vivienda, agua potable, condiciones óptimas de salud, infraestructura o mitigación del efecto de desastres naturales" (PNUMA, 2010).

Por tanto, las alternativas de desarrollo posible para un entorno social determinado están estrechamente vinculadas con la capacidad de los ecosistemas de continuar prestando los bienes y servicios ambientales suficientes para sustentar el modelo de desarrollo en cuestión. Este análisis resulta más complejo porque los esfuerzos deben estar dirigidos a garantizar el principio de sostenibilidad generacional, es decir, no solo en beneficio de las generaciones presentes, sino también de las generaciones futuras.

De modo que el tránsito hacia la sostenibilidad requiere que en el diseño y ejecución de procesos de desarrollo, en particular cuando de desarrollo local se trata, se cumplan con principios básicos, tales como el enfoque integral y sistémico del desarrollo, es decir, teniendo en cuenta su dimensión económico-productiva, institucional, socio-cultural y ambiental, así como el fortalecimiento de la institucionalidad y el fomento de la participación autogestionaria (Guzón et al., 2011). Este tipo de procesos debe tener en cuenta en su diseño e implementación los principios del EE como elemento clave de la dimensión ambiental del desarrollo. 
Se considera que a pesar de que el EE ha

sido adoptado hace varios años por la CDB y de la existencia de guías operacionales y propuestas de implementación práctica, aún se requiere demostrar, en diversos escenarios de gestión territorial, su eficacia como estrategia de conservación y desarrollo sostenible. En este sentido, es necesario generar mayores capacidades técnicas y políticas para su aplicación efectiva (Andrade et al., 2011).

Así es que a pesar de que a nivel internacional se ha reconocido la necesidad de lograr una gestión más integrada de "lo ambiental" lo cierto es que las medidas adoptadas hasta el momento no resultan suficientes, pues "cerca del $60 \%$ de los ecosistemas del mundo están degradados o son utilizados en formas insostenibles" (PNUMA, 2010). Es decir, nos encontramos ante problemáticas que el derecho ambiental no ha podido frenar o prevenir, incluso, pudiera ser que no fuera suficiente para prevenirlas o ponerles freno en el tiempo razonable o necesario para detener o evitar la degradación de los ecosistemas.

Asimismo, frente a todo ello han surgido nuevas propuestas como la del derecho de la sostenibilidad, que tiene tres partes: I) los aspectos sociales e institucionales: la gobernanza, cómo nos regulamos, cómo establecemos mecanismos de inclusión, cómo evitamos la marginación social; II) el derecho económico: cómo generamos y distribuimos la riqueza; y III) el derecho ambiental: cómo nos comportamos con el medio ambiente. Estos tres órdenes de derecho se encuentran en cualquier Estado y tienen un fundamento soberano, es decir, forman parte del Derecho del Estado y tienden a resolver problemas locales o de grupo en sentido clásico. La cuestión es que todos estos órdenes jurídicos están abordando situaciones para las que ya no es suficiente el fundamento soberano para resolver un problema de grupo, sino que se ve implicada la especie como sustrato social, como fundamento legitimador de ese derecho, ya que lo que tienden a resolver son problemas de carácter global, a hacer reales los Objetivos del Milenio; esto es, lo que sería el nuevo derecho de la sostenibilidad. El derecho de la sostenibilidad es un derecho pensado en términos de especie y en términos de resolver problemas globales. Trae parte de la estructura clásica de los órdenes jurídico, social, económico y ambiental, que son propios de los Estados soberanos, pero desborda ese ámbito. Es una rama de derecho emergente en transformación, que ha arrastrado tras de sí al derecho ambiental, las cuestiones sociales y económicas, en la búsqueda de una sociedad global. En consecuencia, lo ambiental forma parte de un todo más grande (Real, 2008).

No obstante, se plantea que "las mayores dificultades no están en lograr consensos en los niveles internacionales o globales sino que, en las áreas del Estado Nacional es donde falta la voluntad para avanzar hacia nuevos compromisos y para concretar 
en decisiones políticas los pactos firmados" (Méndez, 2007). Esto se relaciona con el reconocimiento de las obligaciones asumidas por los Estados en virtud del Derecho internacional hacia lo interno de los marcos legales nacionales, así como con la capacidad de ejecución y cumplimiento de las regulaciones nacionales.

De modo que, sin negar la necesidad de regulación en relación a determinadas esferas específicas de protección ambiental, lo cierto es que se impone la necesidad de lograr que no sean los temas ambientales otro sector dentro de la sociedad, sino que se visualicen e incorporen enfoques más sistémicos que propicien la integración de lo ambiental dentro de las políticas de desarrollo de los países, tanto a escala nacional como territorial y local. Una vía de hacerlo pudiera ser la incorporación efectiva del EE y los principios que lo integran en las políticas públicas y sus respectivos instrumentos operacionales. En función de ello resulta atinado evaluar los retos y desafíos de los ordenamientos jurídicos nacionales para propiciar el tránsito hacia el desarrollo sostenible.

\section{EVOLUCIÓN DE LOS ORDENAMIENTOS JURÍDICOS NACIONALES PARA LA PROTEC- CIÓN DEL MEDIO AMBIENTE EN LA REGIÓN DE AMÉRICA LATINA Y EL CARIBE}

El derecho ambiental, o lo ambiental como objeto de tutela jurídica por el derecho, no se limita a la relación del hombre con el hombre, sino que incluye el tema de la relación del hombre con el entorno, de modo que cambia la situación de un sistema binario a uno triangular, de ahí que se considere que los instrumentos tradicionales del Derecho no siempre resulten suficientes para dar respuesta a la complejidad que implica la tutela jurídica del medio ambiente (Real, 2008).

Lo anterior ha determinado que el campo del derecho ambiental conformado a partir de las tradicionales instituciones jurídicas que obedecen a una concepción represiva del Derecho, ya sea por la vía administrativa, civil o penal, se direccione hacia una concepción preventiva que ha tenido como instrumento más representativo la Evaluación de Impacto Ambiental, aunque ello no implica la exclusión de los instrumentos de comando y control, sino más la complementariedad entre unos y otros.

Esta misma concepción de complementariedad es la que se manifiesta en relación a otros aspectos que el Derecho debe estar regulando en su acometido de protección ambiental, tales como las técnicas de mercado y la internacionalización de costos y la participación ciudadana en la adopción de decisiones relacionadas con temas ambientales.

Se reconoce que "la brecha entre el trabajo analítico y normativo y el nivel operacional es cada vez mayor. El principal foco de atención y de acción se está alejando del desarollo de normas y políticas para centrarse en su aplicación en todos los países" (PNUMA, 2007). Es por ello que en el III Programa de 
Montevideo* se reconoce la problemática relacionada con la aplicación y cumplimiento del derecho ambiental en tanto identifica como primer objetivo estratégico el siguiente: lograr una aplicación, cumplimiento y vigilancia efectivos del derecho ambiental.

Debido a esta situación el debate en torno a la eficiencia y la eficacia de la legislación ambiental, en tanto instrumento de la política y la gestión ambiental, ha ocupado múltiples escenarios en la región de América Latina y el Caribe. En tal sentido, se considera como figura cúspide sobre este debate al Dr. Raúl Brañes Ballesteros, quien plantea que "existe una percepción generalizada en orden a que la legislación ambiental -en todas partes del mundo- tiene un bajo nivel de aplicación cuyo análisis a los efectos metodológicos se distingue entre eficiencia y eficacia" (Brañes, 1994). A estos efectos, el mismo autor considera que "la eficiencia se refiere al grado de idoneidad de una norma jurídica para alcanzar los objetivos que se tuvieron en cuenta al momento de su expedición y la eficacia al grado de acatamiento de una norma jurídica por sus destinatarios" (Brañes, 1994 y 2006).

Este mismo autor plantea que

* Los Programas para la Revisión y Examen Periódico del Derecho Ambiental, conocidos como Programas de Montevideo, guían el actuar del programa de Naciones Unidas para el Medio Ambiente en materia de Derecho Ambiental. El Programa de Montevideo I fue aprobado en el año 1981; Montevideo II, en 1993; Montevideo III, en 2001, y Montevideo IV, en 2009, y estará en vigor para el decenio 2010-2020. http://www.pnuma.org/deramb/ documentos/VIProgramaRegional/2\%20DERECHO\%20 Y\%20GOBERNANZA/2\%20Andrea\%20PNUMA\%20y\%20 el\%20derecho\%20amb.pdf la eficacia y la eficiencia de la legislación ambiental son como las dos caras de una misma medalla, pero distinguir una y otra es importante para fines de análisis porque permite identificar con mayor precisión las razones que están detrás de la falta de aplicación, que muchas veces son su propia falta de idoneidad (Brañes, 2000).

También plantea que

existe una percepción generalizada en orden a que la legislación ambiental tiene un bajo nivel de aplicación... es importante precisar que los factores de ineficiencia de la legislación ambiental están presentes en los sistemas jurídicos de la región... y contribuyen a crear un cuadro de ineficacia de la legislación ambiental, sin embargo, la ineficacia de la legislación ambiental no se explica solo por razones de ineficiencia. Si así fuera -precisa este autor- todo el problema se reduciría a mejorar técnicamente dicha legislación (Brañes, 2006).

En este mismo artículo, Brañes enuncia como factores que influyen en la ineficiencia de la legislación ambiental tanto la falta de desarrollo de la legislación como el enfoque equivocado que asume para el tratamiento de los asuntos ambientales lo que se manifiesta en la falta de presencia de la idea del desarrollo sostenible en el sistema jurídico en general y en particular en la legislación económica; la carencia de instrumentos apropiados para su aplicación, particularmente en lo relacionado con los instrumentos de naturaleza 
preventiva; la falta de consideración de las cuestiones sociales y naturales involucradas en los asuntos ambientales y la heterogeneidad material y estructural de la legislación ambiental (Brañes, 2006).

En relación a la eficacia, identifica como factores esenciales los siguientes: la insuficiente valoración social de la legislación ambiental por sus destinatarios, lo que muchas veces incluye el desconocimiento de esta; y las deficiencias que presentan las instituciones encargadas de aplicar administrativa y judicialmente la legislación ambiental (Brañes, 2006).

Siguiendo a Brañes Ballesteros, otros autores han planteado que

los problemas de aplicación de la ley se derivan, en muchos casos, del hecho de que las normas jurídicas están formuladas desconociendo los fenómenos o las situaciones que se pretenden regular o los aspectos científicos, tecnológicos, sociales o económicos que están involucrados en una problemática determinada (Cancino, 2006).

\section{Plantea otro autor que}

no es la dispersión, entendida como profusión de normas dictadas por diferentes órganos de gobierno, el talón de Aquiles del derecho ambiental; es la falta de una mayor y profunda elaboración teórica de esta ciencia, que nos permita dictar nor- mas jurídicas eficaces y eficientes, es la falta de voluntad política de los gobiernos para dictar las normas y luego ejecutar las acciones que aseguren su cumplimiento (Caraballo, 2002).

Por último, sostiene este autor que

la eficacia de la norma de Derecho Ambiental, está relacionada con su capacidad de ser reflejo de la doctrina y la práctica jurídica vinculadas a la conservación de la diversidad biológica... ser eficaz depende de una adecuada percepción del ambiente, esto es, de que opere sobre la base de que el ambiente constituye un acoplamiento de subsistemas ecológicos funcionalmente interdependientes, constituidos a su turno, por factores dinámicamente interrelacionados (Caraballo, 2002).

Este mismo autor considera que

la actitud de la población frente a la norma es el resultado de la costumbre, del grado de instrucción, de los intereses económicos y sociales, las necesidades materiales y espirituales de dicha población... que serán los que determinen que una norma jurídica sea eficiente.

Por otra parte, otro autor plantea que "existe una inflación normativa, que obedece más a respuestas ante situaciones coyunturales, que a un desarrollo coherente y ordenado de las políticas ambientales que se han formulado" (Mora, 2002). Ahora bien, importante 
es precisar que la eficiencia de la legislación no debe evaluarse de forma aislada en una norma jurídica o en un precepto de una disposición jurídica determinada, sino de forma integrada en el conjunto de disposiciones jurídicas que como sistema conforman un ordenamiento jurídico.

No obstante, en el proceso de construcción del acto normativo, debe tenerse en cuenta lo que plantea Rey Santos (2006)* en el sentido de que "es un espejismo pensar que una nueva ley resolverá problemas que en realidad están determinados por la realidad material -modelos económicos, desarrollos tecnológicos, patrones de producción- que dificultan o incluso neutralizan la aplicación de la ley". Por esta razón, refiere Rey Santos, que "aplicar una ley no se basa por supuesto en dictar más leyes que le complementen aunque esto puede ser necesario, es un fenómeno más amplio, que demanda de voluntad política y recursos materiales, financieros y humanos", pues de lo contrario lo que se genera en muchos casos, según precisa este mismo autor, "es el dictado de una norma tan o más insuficiente que la preexistente e igualmente condenada a su incumplimiento".

Así, pues, se ha planteado además que

la existencia formalmente determinada de la norma jurídica... contiene la posibilidad

* Comunicación personal con el autor: Rey S., O. Director de Medio Ambiente. Ministerio de Ciencia, Tecnología y Medio Ambiente. La Habana, 2006. de contradicción entre la norma jurídica y la realidad. La propia eficacia de la norma jurídica depende en gran medida de la exactitud con que se reflejan en ella las necesidades y las leyes objetivas del desarrollo social (Zhidkov et al., 1980).

En todo caso, esa realidad material es la base inexcusable sobre la cual debe diseñarse el contenido de la disposición jurídica según sea el objetivo que pretende lograrse con tal regulación, pues, como plantea otro autor, es un error la

idea altamente generalizada de que el Derecho es solo una larga fila de normas jurídicas dictadas caprichosamente... y que pueden resultar eficaces y válidas aunque nada tengan que ver con la existencia material de la sociedad a la que supuestamente están dirigidas (Fernández, 2004).

Así es que la disposición jurídica tiene que descansar en la sociedad, ser reflejo de los intereses sociales y condiciones materiales que le dan origen, es decir, debe ser expresión de los intereses comunes derivados del régimen material de producción existente en cada época. $Y$, por tanto, debe establecer regulaciones cumplibles en un contexto determinado que no se traduzcan en regulaciones inviables, más catálogo de aspiraciones y principios que auténticos textos legales (Rey, 2011). En esta misma línea de pensamiento, plantea otro autor que no basta con dictar normas exigentes para cumplir los objetivos de protección que nos animan, pues por más 
que se establezcan determinados requisitos en la legislación, no van a poder ser cumplidos si el sistema productivo existente no puede lograrlo.

Otro autor consultado plantea que "la eficacia no es una condición de validez de la norma, es decir, de su existencia como tal norma: una norma es válida, aunque no se aplique porque no la acepten sus destinatarios o porque los jueces no velen por su observancia... La eficacia de las normas depende del grado de aceptación social y del grado de coacción protectora; si faltan al mismo tiempo ambas condiciones..., la norma sufrirá una absoluta ineficacia, convirtiéndose en norma en desuso (Soriano, 2000).

En ocasiones sucede que existen en el ordenamiento jurídico normas válidas, en tanto han sido promulgadas por los órganos y procedimientos competentes, si no han sido expresa o presuntamente derogadas por otra norma, ni entran en contradicción con una de carácter superior jerárquica o de preferente aplicación en el ordenamiento jurídico, pero su absoluta falta de aplicación conlleva a su ineficacia, caso en el cual se considera que "una norma en desuso no existe como tal norma" (Soriano, 2000). El "desuso" de la norma puede estar dado por su obsolescencia-es decir, la variación de las condiciones y aspectos que regula aunque en determinado momento si se ajustaba a tales condiciones, o porque nunca reconoció adecuadamente en el texto de la ley las realidades del fenó- meno o proceso objeto de regulación jurídica.

Se plantea que

la eficiencia de una norma jurídica implica el logro del objeto, propósito o fin de una norma jurídica o de una ley. Esta noción es distinta de la de eficacia. Una ley puede ser eficaz, ser obedecida por los sujetos de la misma, sin que su objeto haya sido alcanzado (Esquivel, 1972).

David G. Víctor plantea que "en la mayoría de los estudios, en particular los realizados por abogados, se equipara la 'efectividad' con el 'cumplimiento', pero ese enfoque rara vez es atinado". Considera este autor que "la 'efectividad' es una medida del impacto de la ley en el comportamiento; las leyes más efectivas producen mayores efectos en la conducta infractora; las menos efectivas, ninguno". Siendo así -en criterio de este autor que distingue entre "efectividad" y "cumplimiento"-, evaluar la "efectividad" de las leyes implica no solo tener en cuenta sus repercusiones en el comportamiento, sino también su costo social, mientras que el "cumplimiento" se refiere simplemente a la "observancia de la ley" (CCA, 1999).

En tal sentido, otro autor consultado aborda esta problemática desde la perspectiva de la realización del derecho, y al respecto plantea que esta incluye en términos generales "tanto la aplicación estatal u oficial de las normativas jurídicas, como el cumplimiento 
espontáneo de las mismas por parte de los depositarios de dichas normas" (Fernández, 2004). Esto, teniendo en cuenta que en algunos casos la aplicación estatal no se limita a la ejecución coactiva de la norma ante la comprobación de una conducta infractora, por ejemplo, sino que el cumplimiento voluntario de la norma requiere de la intervención estatal, como es el caso de la tramitación de una Licencia Ambiental.

No obstante, hace notar que el derecho se realiza "sobre todo, cuando sus fines son cumplidos, cuando sus objetivos ordenadores de la conducta social quedan satisfechos porque los depositarios de esas normas, con su conducta natural se ajustan a las mismas, acomodan sus actos a las prescripciones del Derecho" (Fernández, 2004). Este sería el caso en que se constate la efectividad de la legislación ambiental, ya sea por la evolución de la calidad del medioambiente y los efectos en la salud de las personas o porque el uso de los ecosistemas no afecta su capacidad para continuar prestando bienes y servicios ambientales. No obstante, en tal sentido es importante hacer notar que la realización del derecho en este supuesto implica necesariamente que la formulación de la disposición jurídica $-\mathrm{y}$ las vías, procedimientos e instrumentos previstos por esta- se corresponda realmente con los objetivos que se pretenden alcanzar con su diseño, pues de lo contrario estaríamos ante un caso de aplicación de la legislación, incluso de forma voluntaria, pero que no permite obtener los objetivos deseados, debido a las deficiencias de diseño de la legislación en cuestión.
Más allá o con independencia de si la legislación que se diseña es adecuada para obtener los objetivos políticos previstos, lo cierto es que se reconoce en sentido general que "la capacidad de gestión de las instituciones ambientales en la región se ve limitada... por la debilidad política para hacerlas cumplir" (PNUMA, 2010).

Se identifican como retos en cuanto a la aplicación y cumplimiento de la normativa ambiental los siguientes: mejorar la distribución de competencias a nivel gubernamental, fortalecer y consolidar los mecanismos de participación ciudadana, mejorar la transparencia ambiental, exigir mayor consistencia de las políticas ambientales y económicas, articular y consolidar la educación legal-ambiental y los avances en el conocimiento científico y de las posibilidades tecnológicas (PNUMA, 2007).

Esta complejidad se incrementa cuando estamos hablando de ordenamientos jurídicos que deben regular procesos de desarrollo que en su diseño y aplicación tengan en cuenta el EE como alternativa que puede posibilitar el tránsito hacia la sostenibilidad. Siendo este un reto que se agrega a las ya conocidas dificultades relacionadas con la aplicación y cumplimiento del derecho ambiental que se ha venido produciendo hasta el momento. De ahí que resulte oportuno abordar los retos que el EE representa para los ordenamientos jurídicos nacionales de los países de la región. 


\section{EL ENFOQUE POR ECOSISTEMAS COMO RETO PARA LOS ORDENAMIENTOS JURÍDI- COS NACIONALES}

La aplicación del EE, visto desde su concepción general como desde sus principios de forma individualizada, implica necesariamente y, en mayor o menor medida, el apoyo o respaldo en políticas, legislación y sus respectivos instrumentos operacionales como vías de implementación del marco político y legal. Por eso se plantea, refiriéndose en este caso a las cuencas hidrográficas -como un importante ecosistema en torno al cual, bajo diversas modalidades, se ha promovido el EE-, que "es necesario articular mejor el desarrollo de políticas y de legislación, las herramientas económicas y el desarrollo de infraestructura, con la gestión de las cuencas y los ecosistemas aportantes" (Guerrero et al., 2007).

En tal sentido, es importante hacer notar que la referencia a política y legislación no se refiere únicamente a lo ambiental, sino más bien las políticas del país con implicación en el desarrollo. Este enfoque se corresponde con la necesidad de que lo ambiental pase de ser un sector a estar incorporando en las políticas de desarrollo, pues se considera que "la productividad de los ecosistemas depende de las políticas que se apliquen, incluidas las relativas a inversiones, comercio, subsidios, impuestos y regulación" (MEA, 2005).

Es importante tener en cuenta que para lograr el desarrollo sostenible es fundamental integrar las preocupaciones ecológicas en las políticas de desarrollo pues los problemas ambientales y de desarrollo están vinculados por procesos sociales y ambientales que atraviesan fronteras temáticas, institucionales y geográficas, que exigen un enfoque sistemático, sostenido, integrado y coherente en el que participen diferentes administraciones a distintas escalas (PNUMA, 2007).

En tal sentido, se considera que "la gestión de recursos comunes y sistemas complejos puede requerir un enfoque flexible con un conjunto amplio de herramientas de gobiernos a distintos niveles" (Dietz et al., 2003). De ahí que se identifique como un reto para el Manejo Integrado de Aguas y Áreas Costeras en los países de la región, en tanto modalidad del EE, el fortalecimiento y desarrollo de los instrumentos ambientales para que en su implementación contribuyan de forma efectiva al desarrollo sostenible (PNUMA ALC, 2011).

Se considera que los principios del EE que mayor relación guardan con el marco político y legal son los siguientes:

- Un primer bloque de principios cuya aplicación implica prever formas de participación de las comunidades indígenas y locales en la adopción de decisiones, de modo que estas no vengan a ser un nivel inferior de consulta sino más bien un actor importante del proceso que puede estar aportando al análisis en cuestión 
toda vez que es parte de este, argumentos con los que guardan relación el Principio 1: La elección de los objetivos de la gestión de los recursos de tierras, hídricos y vivos debe quedar en manos de la sociedad; el Principio 11: En el enfoque ecosistémico deberían tenerse en cuenta todas las formas de información pertinente, incluidos los conocimientos, las innovaciones y las prácticas de las comunidades científicas, indígenas y locales, y el Principio 12: En el enfoque ecosistémico deben intervenir todos los sectores de la sociedad y las disciplinas científicas pertinentes.

- Otro principio cuya aplicación requiere de la adecuación o diseño de marcos institucionales que permitan la adopción de decisiones lo más cercano posible a donde se da la relación entre los recursos naturales y las comunidades indígenas y locales, se trata del Principio 2: La gestión de los recursos naturales debe estar descentralizada al nivel apropiado más bajo.

- Otro principio que se refiere a la necesidad de prever la valoración económica de bienes y servicios ambientales, así se pueden planificar los diferentes mecanismos económicos e incentivos como herramientas que también pueden contribuir a la conservación de los ecosistemas, a cuyo efecto se necesita que los ordenamientos jurídicos nacionales hagan las adecuaciones correspondientes. Se trata del Principio 4: Dados los posibles beneficios derivados de su gestión, es necesario comprender y gestionar el ecosistema en un contexto económico. Este tipo de programa de gestión de ecosistemas debería: I) disminuir las distorsiones del mercado que repercuten negativamente en la diversidad biológica; II) orientar los incentivos para promover la conservación y la utilización sostenible de la diversidad biológica; y III) procurar, en la medida de lo posible, incorporar los costos y los beneficios en el ecosistema de que se trate.

Siendo así, los mayores retos que encuentran los autores para la incorporación del EE en la región se identifican en relación al marco institucional y los procesos de descentralización que están ocurriendo en varios países, la participación ciudadana como herramienta clave del proceso de adopción de decisiones y la internalización de los costos mediante una adecuada valoración económica de bienes y servicios ambientales que prestan los ecosistemas y el diseño de incentivos que promuevan la conservación de estos bienes y servicios.

\subsection{Marco institucional flexible}

Un marco institucional que en su diseño no excluya los intereses sectoriales sino que en su funcionamiento contribuya a la integración y operación coordinada en función de objetivos comunes que requieren ser abordados de forma sistémica. Así es que algunos de los asuntos de manejo son comunes para las cuencas hidrográficas y las zonas costeras de modo que requieren de enfoques integrados para su gestión. Siendo así, de lo que se 
trata es de "hacer prevalecer el enfoque ecosistémico sobre el enfoque sectorial" (CITMA, 2009).

Por ello, se considera que un aspecto clave en la implementación de los enfoques integrados en la gestión de cuencas hidrográficas y zonas marino-costeras es la coordinación intersectorial entre autoridades y niveles con competencias para la dirección de estos ecosistemas. En relación con este tema, se identifica como un avance en la región de América Latina y el Caribe la existencia de algunas experiencias positivas de coordinación intersectorial en diferentes niveles para la gestión integrada de zonas costeras y cuencas hidrográficas. No obstante, al respecto también se identifica el reto de favorecer el fortalecimiento de estos mecanismos de coordinación intersectorial con una visión integral de la gestión de los ecosistemas (PNUMA ALC, 2011).

Otro aspecto relacionado con un marco institucional que pudiera favorecer la adopción del EE es la descentralización hacia lo local ${ }^{*}$ en el sentido de que se propicie el "diseño y puesta en funcionamiento efectivo de mecanismos de gobernanza participativos y descentralizados, que logren una gestión ecosistémica justa y equitativa, así como una efectiva gestión del conocimiento desde todas sus fuentes" (Andrade et al., 2011). En tal sentido, se plantea que

\footnotetext{
* Esto corresponde con el Principio 2 del EE que establece que "la gestión de los recursos naturales debe estar descentralizada al nivel apropiado más bajo".
}

una descentralización eficaz requiere una delegación adecuada de la autoridad, lo cual implica que los interesados directos gocen tanto de la oportunidad de asumir la responsabilidad como de la capacidad para aplicar las medidas apropiadas, y es necesario que esté apoyada por marcos normativos y legislativos que lo faciliten (Andrade et al., 2011).

Se considera que "es más probable que las medidas destinadas a conservar los recursos naturales tengan éxito si se otorga a las comunidades locales la propiedad de los mismos, y si ellas participan en el reparto de beneficios y están involucradas en las decisiones". Del mismo modo que es muchísimo más probable que las comunidades locales conserven los recursos naturales si ellas tienen una influencia real sobre las decisiones en cuanto al uso de los mismos -y si a la postre logran un reparto más equitativo de los beneficios (MEA, 2005).

Importante es tener en cuenta que "una mayor participación de las comunidades indígenas en la toma de decisiones también puede brindar acceso al conocimiento tradicional con respecto al funcionamiento de los sistemas naturales para ayudar a diseñar formas más eficientes de protegerlos" (MEA, 2005).

Al respecto se considera que "se deben aumentar más las oportunidades de las poblaciones locales para incorporar nuevas tecnologías y usos del suelo alternativos y sostenibles, incluyendo actividades no agrícolas generadoras de ingresos que puedan atenuar 
las presiones sobre el ambiente" (PNUMA, 2010).

En cuanto al marco legal e institucional para el Manejo Integrado de las Zonas Costeras bajo la concepción de EE de Manejo Integrado de Aguas y Áreas Costeras, se identifica como aspectos que significan avances en la región el hecho de que todos los países han adoptado los Acuerdos Multilaterales de Medio Ambiente y cuentan con autoridad ambiental con competencias para la gestión de los ecosistemas marino-costeros, sin embargo, en algunos mínimos casos resulta poco claro. No obstante, se identifica como retos en este sentido el diseño y establecimiento de mecanismos legales o plataformas que faciliten la coordinación entre instituciones, sectores y niveles de gobierno y crear o fortalecer las estructuras institucionales de los países para la gestión de los ecosistemas costero-marinos (PNUMA ALC, 2011).

\subsection{Participación ciudadana en la toma de decisiones}

Importante es hacer notar que la participación ciudadana en relación a temas relacionados con lo ambiental ha sido objeto de análisis en el marco de diferentes Acuerdos Ambientales Multilaterales. En tal sentido es importante resaltar el Principio $10^{*}$ de la De-

* El Principio 10 de la Declaración de Río sobre Medio Ambiente y Desarrollo establece lo siguiente: "El mejor modo de tratar las cuestiones ambientales es con la participación de todos los ciudadanos interesados, en el nivel que corresponda. En el plano nacional, toda persona deberá tener acceso adecuado a la información sobre el claración de Río sobre Medio Ambiente y Desarrollo que se pronuncia por la adopción de medidas que permitan el acceso a la información ambiental y a la justicia para dirimir conflictos ambientales, ya sea por la vía administrativa, civil o penal, y a la participación en la adopción de decisiones ambientales, como tres derechos denominados "procedurales" que garantizan el ejercicio del derecho a un medio ambiente sano.

Ahora bien, en tanto en el Enfoque por Ecosistemas coloca a la gente y el uso de los recursos naturales en el punto de partida de la toma de decisiones, el derecho debe prever mecanismos de participación adecuados en los procesos de gestión pública y adopción de decisiones, del mismo modo que deben preverse mecanismos adecuados para la gestión o la solución de los conflictos sobre uso de recursos naturales que pueden generarse como parte del propio proceso de definición de prioridades de usos y, visto a más largo plazo, de decisiones relacionadas con el desarrollo. Por tanto, la participación que se requiere para promover el EE no se limita a la consulta pública asociada al Proceso de Evaluación de Impacto Ambiental de un Proyecto de obra o actividad, aunque puede incluirla.

medio ambiente de que dispongan las autoridades públicas, incluida la información sobre los materiales y las actividades que encierran peligro en sus comunidades, así como la oportunidad de participar en los procesos de adopción de decisiones. Los Estados deberán facilitar y fomentar la sensibilización y la participación de la población poniendo la información a disposición de todos. Deberá proporcionarse acceso efectivo a los procedimientos judiciales y administrativos, entre estos el resarcimiento de daños y los recursos pertinentes". 
Este es un elemento importante en tanto se advierte que en muchos casos la participación ciudadana no pasa de ser un mero requisito a cumplir por el actor económico (público o privado) antes del emprendimiento de una actividad con impactos ambientales, generando entre la población reacciones adversas que muchas veces generan conflictos sociales que llegan a comprometer las bases de la sociedad (PNUMA, 2010).

Por ello es tan importante fortalecer y consolidar los mecanismos de participación ciudadana en tanto representan "uno de los elementos cruciales para hacer funcionar el aparato del sistema ambiental, así como para el logro de normas ambientales eficientes y efectivas, que es en suma lo que debería esperarse como corolario de dicho proceso" (PNUMA, 2010). En tal sentido se hace notar además que "es fundamental honrar los principios del enfoque ecosistémico referidos a la participación de los actores locales en la toma de decisiones" (Guerrero et al., 2007).

No obstante, consideramos que la participación ciudadana es un tema medular no solo en cuanto a las problemáticas ambientales, sino más bien y por sobre todas las cosas para el desarrollo de los países en tanto algunos autores plantean que "la fuerza principal de una nación no reside en las reservas de petróleo, hierro, uranio, carbón, agua, esto es, el capital natural de un país, sino en su capital social, en el sentido de la capacidad de crear que poseen los individuos que pueblan un determinado territorio" (Fung, 2000).

Así es que se plantea que

los proyectos de desarrollo local deben concebirse como un proceso participativo y escenario de aprendizaje en el que se manifieste una real participación de los actores involucrados y de la población beneficiaria en los espacios de toma de decisiones, para ello se requiere del reconocimiento y la conciliación de la diversidad de actores, intereses y roles (Guzón et al., 2011).

Es por ello que los modelos de manejo integrado de determinados ecosistemas que en su diseño y aplicación observan principios del EE -como es el caso del Manejo Integrado de las Zonas Costeras, el Manejo Integrado de las Cuencas Hidrográficas, la Gestión Integrada de Recursos Hídricos y el Manejo Sostenible de Tierra- se pronuncian por la participación de los ciudadanos en el diseño y conducción de este tipo de procesos.

En tal sentido es importante hacer notar que se plantea que

es deseable que los sectores más sensibles y responsables con el compromiso ambiental evolucionen de una posición 'ambientalista' hacia la consideración de la multidimensionalidad del desarrollo y de las decisiones que lo hacen posible, rompiendo el estigma de la parcialidad que a veces sobredimensiona los objetivos 
ambientales sin tomar en cuenta legítimas aspiraciones de uso y aprovechamiento sostenible de los recursos naturales en acciones de desarrollo socioeconómico (PNUMA ALC, 2010).

\subsection{Internalización de costos ambientales}

Se considera que este es un tema absolutamente importante en tanto "es improbable que la protección de los servicios de la naturaleza se convierta en una prioridad mientras los que los usan los sigan percibiendo como servicios gratuitos e inagotables" (MEA, 2005). Por tanto, se requiere que las políticas, la legislación y la adopción de decisiones tengan en cuenta los costos naturales como parte de las decisiones de tipo económico, pues solo de esta forma se estaría transitando el camino hacia la sostenibilidad.

Para ello resulta importante "reconocer como activos los bienes y servicios que proporciona la combinación de ecosistemas" (Smith et al., 2007) de modo que en el análisis asociado a la adopción de decisiones se evalúe bajo un criterio de costo-efectividad no solo el proyecto de obra o actividad en cuestión, sino también y por sobre todas las cosas lo que implica en relación a la capacidad del ecosistema de continuar prestando bienes y servicios ambientales. Por ejemplo, hay que tener en cuenta qué implicaciones económicas genera la afectación al bosque de manglar y a los bienes y servicios que este presta, tales como reducción de la vulnerabilidad costera y atenuación del oleaje, pro- ducción primaria de nutrientes para la cadena trófica de especies marinas de la zona del litoral, además de productos no madereros como mieles y fibras (Guzmán et al., 2006).

Ahora bien, la valoración económica de los bienes y servicios ambientales que prestan los ecosistemas constituye un elemento clave no solo para una adecuada adopción de decisiones, sino también para el diseño de incentivos económicos que promuevan o apoyen su conservación. Por consiguiente, se plantea que

es relevante la continuación en los esfuerzos de desarrollo e implementación de incentivos económicos que apoyen la conservación y el uso sostenible de los ecosistemas, y que consideren los aspectos sociales y culturales de quienes habitan los territorios (PNUMA, 2010).

En este orden de ideas, es importante tener en cuenta también que el Cuarto Programa para la Elaboración y el Examen Periódicos del Derecho Ambiental se pronuncia por la promoción del cumplimiento voluntario y los incentivos económicos. En tal sentido, define dentro sus estrategias para aumentar la eficacia del derecho ambiental la de "identificar y promover enfoques, instrumentos y mecanismos innovadores que potencien la eficacia del Derecho Ambiental", dentro de los cuales se encuentran el etiquetado ecológico, la certificación, las tasas por contaminación y los impuestos sobre los recursos naturales, por citar ejemplos (PNUMA, 2009). 
En cuanto al marco legal e institucional para el Manejo Integrado de las Zonas Costeras bajo la concepción de EE de Manejo Integrado de Aguas y Áreas Costeras, se identifica como aspectos que significan avances en la región el hecho de que algunos países tienen incentivos para el cumplimiento voluntario de estándares ambientales y que existen experiencias de establecimiento de regulaciones legales y su aplicación sobre la valoración de los servicios ambientales, lo que hicieron notar los participantes de países como Costa Rica, República Dominicana y México. No obstante, se identifican retos en cuanto a este tema, entre los cuales se encuentran la necesidad de fortalecer el establecimiento y la aplicación de instrumentos de autogestión para el cumplimiento ambiental y de desarrollar o fortalecer el marco legal para la valorización de los servicios ambientales (PNUMA ALC, 2011).

\section{CONCLUSIÓN}

Se reconoce a nivel internacional y en las políticas públicas de los países la necesidad de propiciar la aplicación del Enfoque por Ecosistemas y sus principios como vía para disminuir las brechas entre lo ambiental y lo económico, así como el importante rol que desempeña el ser humano en estos procesos. No obstante, en la práctica de las instituciones públicas, sus herramientas de gestión y experiencias de funcionamiento no se manifiestan de forma generalizada resultados palpables y medibles en este sentido que requiere llevar a una reflexión e inclusión mucho más efectivas.
Continúa siendo un reto para la región el diseño de marcos institucionales suficientemente flexibles para posibilitar la coordinación intersectorial en diferentes niveles de los gobiernos de modo que no se excluyan las competencias, sino que operativamente se fortalezcan las actuaciones en las esferas o problemáticas de interés común de actuación.

Al mismo tiempo también constituye un reto que se garantice la participación de los ciudadanos de forma activa no solo en los temas de interés ambiental y asociados a los procesos específicos de toma de decisiones que conciernen a lo ambiental, sino más bien de forma autogestionaria en los procesos de desarrollo.

También es un reto la valoración de los servicios de los ecosistemas -incluyendo el desarrollo y aplicación de los marcos normativos adecuados- de modo que se potencie la aplicación de incentivos y otros instrumentos económicos que promuevan la aplicación y cumplimiento de la legislación que, sin excluir el rol de instrumentos tradicionales de comando y control, estén más dirigidos a internalizar los costos ambientales.

Por último, un reto con énfasis particular es la potenciación de políticas públicas que promuevan el desarrollo local, así como el rol que corresponde jugar a las comunidades indígenas y locales en la definición de las líneas de desarrollo prioritarias de acuerdo a los usos posibles de los recursos naturales, 
teniendo en cuenta la conexión entre el modelo de desarrollo a adoptar y la capacidad de los ecosistemas para continuar prestando bienes y servicios ambientales.

\section{REFERENCIAS}

\section{Libros}

Andrade, A., Arguedas, S., Vides, R. (2011). Guía para la aplicación y monitoreo del Enfoque Ecosistémico, CEM-UICN, UNESCO-Programa MAB, Cl-Colombia, ELAP-UCI, FCBC.

Brañes B., R. (1994). Manual de derecho ambiental mexicano. Fundación Mexicana para la Educación Ambiental. México: Fondo de Cultura Económica.

Brañes B., R. (2000). El Acceso a la justicia ambiental en América Latina. Asociación Latinoamericana de Derecho Ambiental. México.

Brañes, B., R. (2006). Los mecanismos para la aplicación del Derecho para el desarrollo sostenible en América Latina en la Fundación del Derecho Ambiental en América Latina. Programa de Naciones Unidas para el Medio Ambiente. Oficina Regional para América Latina y el Caribe. PNUMA.

Brito, G., L. (2010). Instituciones nacionales para la gestión integrada de aguas y áreas costeras. Oficina Regional para América Latina y el Caribe del Programa de Naciones Unidas para el Medio Ambiente. Memorias del Curso Regional Manejo Integrado de Aguas y Áreas Costeras en América Latina y el Ca- ribe: Teoría y Práctica. Panamá, del 10 al 20 de agosto.

Fernández B., J. (2004). Teoría del Estado y el derecho. Teoría del derecho. Segunda parte. La Habana: Editorial Félix Varela.

Cancino, M., A. (2006). Gestión ambiental y aplicación de la ley en México en la fundación del derecho ambiental en América Latina. Programa de Naciones Unidas para el Medio Ambiente. Oficina Regional para América Latina y el Caribe. PNUMA.

Castillo, B., Federico, A., Quesada L., J. E., Ramallo L., F. E. (2010). Monografías de gobiernos locales. Gobierno y democracia local: La experiencia andaluza y andina. Unión Iberoamericana de Municipalistas. Granada.

Cincin-Saint, B. \& Knecht, R. W. (1998). Integrated costal and ocean management: concepts and practices. Washington DC.

Dietz, T., Ostrom, E. \& Stern, P. C. (2003). The Struggle to Govern the Commons. En Science, 302(5652), 1907-1912 en Programa de Naciones Unidas para el Medio Ambiente. Perspectivas del Medio Ambiente Mundial. GEO 4. Medio Ambiente para el Desarrollo. PNUMA.

Fung, T. (2000). La ciencia política en el tránsito al siglo XXI. La Habana, Cuba: Editorial Félix Varela.

García, F., J. M. \& Rey S., O. (2005). Foros de negociación e instrumentos jurídicos interna- 
cionales sobre medio ambiente y desarrollo sostenible. La Habana: Publicaciones Acuario.

GEO Cuba (2009). Evaluación del medio ambiente cubano. La Habana: CITMA.

Guerrero, F., E. (2007). El enfoque ecosistémico aplicado a la gestión del agua. Una perspectiva desde América Latina. En Á. Andrade Pérez (Ed.), Aplicación del Enfoque Ecosistémico en Latinoamérica. Bogotá, coIombia: CEM - UICN.

Guzmán J., M. \& Menéndez L., M. (2006). El ecosistema de manglar en Cuba. La Habana: Editorial Academia.

Guzón C., A. \& Colectivo de Autores (2011). Cataurito de herramientas para el desarrollo Local. La Habana: Editorial Caminos.

Ipenza P., C. A. (2010). El convenio sobre la diversidad biológica en el Perú - Análisis de su aplicación y avances en el Perú. Lima, Perú: MINAM.

Lubchenco, J. (1994). The Scientific Basis of Ecosystem Management: Framing the Context, Language and Goals. In EcosystemBased Management: Markers for Assessing Progress. UNEP-GPA, 2006.

Millennium Ecosystem Assessment (2005). Ecosystems and Human Well-Being: Biodiversity Synthesis. Washington, D.C.: World Resources Institute.
Miranda Vera, C.E. (2003). "La zona costera como totalidad ambiental. Primera aproximación". Revista de Investigaciones Marinas, 24(1), 66. En B. Y. Monzón. Tesis en Opción del Grado Científico de Doctor en Ciencias en la Universidad de La Habana, 2011.

Mora P., C. (2002). Avances jurídicos y su aplicación en Colombia en De Río a Johannesburgo: Perspectivas del Derecho Ambiental en Latinoamérica. Oficina Regional para América Latina y el Caribe del Programa de Naciones Unidas para el Medio Ambiente. PNUMA-LAC.

Organización de las Naciones Unidas para la Alimentación y la Agricultura (2011). Resumen del estado de los recursos de tierras y aguas del mundo para la alimentación y la agricultura. ¿Cómo gestionar los sistemas en peligro? FAO.

Pacheco, P. y otros (2008). The role informal institution in the use of forest resources in latin american. CIFOR. Forest gorvernance programme, (15).

Programa de las Naciones Unidas para el Medio Ambiente -PNUMA (2007). Perspectivas del medio ambiente Mundial. Geo 4. Medio ambiente para el desarrollo. Dinamarca: Pnuma.

Programa de las Naciones Unidas para el Medio Ambiente -PNUMA (2009). Oficina Regional para América Latina y el Caribe. Perspectivas del Medio Ambiente: América Latina y el Caribe. 
Programa de Naciones Unidas para el Medio Ambiente (2002). Serie de Documentos Legislativos sobre Derecho Ambiental. No. 3, (1995). En M. L. Caraballo, Tesis en Opción del Grado Científico de Doctor en Ciencias en la Universidad de La Habana.

Rey S., O. (2011). Los retos en la implementación del derecho ambiental en Cuba. En Retos y tendencias del derecho ambiental contemporáneo. Organización Nacional de Bufetes Colectivos. La Habana.

Salvador C., M. (2010). "Modelos constitucionales de gobiernos locales y gobernabilidad democrática". En B. Castillo, A. Federico, J. E. Quesada, F. E. Ramallo, Monografías de gobiernos locales. Gobierno y democracia local: la experiencia andaluza y andina. Unión Iberoamericana de Municipalistas. Granada.

Secretaría del Convenio sobre la Diversidad Biológica (2010). Perspectiva Mundial sobre la Diversidad Biológica 3. Montreal.

Smith, M., de Groot, D., Perrot-Maitre, D. \& Bergkamp, G. (2007). Pago: Establecer pagos por servicios de cuencas. Suiza: IUCN, Gland.

Soriano R. (2000). Los caracteres y las categorías de las normas jurídicas. En J. Fernández \& H. L. Pérez. Selección de lecturas de teoría del Estado y el derecho. La Habana: Editorial Félix Varela.

Zhidkov, O., Chirkin, V., Yudin, Y. (1980). Fundamentos de la teoría socialista del Estado y el derecho. Editorial Progreso. Moscú.

\section{Internet}

Barragán Muñoz, J. M. (2005). La gestión de áreas litorales en España y Latinoamérica. Cadiz: Universidad de Cadiz.

Convenio de Diversidad Biológica (CBD) (2000). Decisión Sexta de la Quinta Conferencia de las Partes "Enfoque por Ecosistemas". http://www.cbd.int/decision/cop/default.shtml?id=7148 Visitado en fecha 26 de agosto de 2013.

David, G. V. (1999). Lecciones de la investigación sobre aplicación y cumplimiento de la legislación internacional en materia de medio ambiente en indicadores de la aplicación efectiva de la legislación ambiental. Memoria de un diálogo en América del Norte. Comisión para la Cooperación Ambiental (CCA). http://www.cec.org/Storage/42/3396_indicsp.pdf Visitado en fecha 13 de agosto de 2013.

Esquivel, J. (1972). Nota Bibliográfica: Foundations of Jurisprudence by Jerome Hall. Crítica: Revista Hispanoamericana de Filosofía, 6(16/17), 139-142, Jan.-May. http://www. jstor.org/stable/40154248 Visitado en fecha 26 de agosto de 2013.

Guerrero, E. (2004). Enfoque ecosistémico y corredores: Dos conceptos que promueven una gestión integral de la biodiversidad. En M. Cracco \& E. Guerrero (edi.), Aplicación del Enfoque Ecosistémico a la Gestión de Corredores en América del Sur. Memorias del TaIler Regional, 3 al 5 de junio de 2004. UICN. Quito, Ecuador. http://data.iucn.org/dbtw- 
wpd/edocs/2004-058.pdf Visitado en fecha 31 de julio de 2013.

Informe del Órgano Subsidiario de Asesoramiento Científico, Técnico y Tecnológico acerca de la labor realizada en su Duodécima Reunión. UNEP/CBD/COP/9/2. CBD, 2007. http://www.cbd.int/doc/meetings/sbstta/ sbstta-12/official/sbstta-12-cop-09-02-es. pdf Visitado en fecha 31 de julio de 2013.

Méndez, F. L. (2007). Globalización y medio ambiente. Revista INAFOCAM, 1(1), 23-4, enero. República Dominicana. http://eprints. ucm.es/7555/1/mendez.pdf Visitado en fecha 31 de julio de 2013.

Principios de manejo integrado de aguas y áreas costeras. Proyecto "Construcción de capacidades en el Manejo Integrado de Agua y Áreas Costeras (MIAAC) en América Latina y el Caribe (2010-2012)". Oficina Regional para América Latina y el Caribe del Programa de Naciones Unidas para el Medio Ambiente. http://www.pnuma.org/agua-miaac/ Visitado en fecha 31 de julio de 2013.

Programa de Naciones Unidas para el Medio Ambiente (2009). UNEP/GC.25/11/Add.2. Cuarto Programa para la Elaboración y el Examen Periódicos del Derecho Ambiental (Programa de Montevideo IV). www.unep. org/gc/gcss- $x /$ download.asp?ID=943 Visitado en fecha 26 de agosto de 2013.

Sherman, K. \& Duda, A. M. (1999). An Ecosystem Approach to Global Assessment and Management of Coastal Waters. Marine Ecology Progress Series, 190, 271-287 www.int-res. com/jounals/meps Visitado en fecha 31 de julio de 2013.

United Nations Environment Programme. Global Programme of Action for the Protection of the Marine Environment from Landbased Activities. Ecosystem-based management: Markers for assessing progress. UNEP/ GPA. The Hague, 2006. http://www.unep. org/pdf/GPA/Ecosystem_based_Management_Markers_for_Assessing_Progress.pdf Visitado en fecha 26 de agosto de 2013.

Waltner-Toews, D. \& Kay, J. (2005). The evolution of an ecosystem approach: the diamond schematic and an adaptive methodology for ecosystem sustainability and health. Ecology and Society, 10(1),38 (on line): http://www. ecologyandsociety.org/vol10 Visitado en fecha 31 de julio de 2013.

\section{Otras fuentes bibliográficas}

Real, F., G. El derecho ambiental y el derecho de la sostenibilidad. Oficina Regional para América Latina y el Caribe del Programa de Naciones Unidas para el Medio Ambiente. Memorias del Quinto Programa Regional de Derecho y Políticas Ambientales. Asunción, Paraguay, del 20 al 30 de mayo de 2008.

Memorias del Segundo Programa Regional de Capacitación en Gobernanza Ambiental de las Zonas Costero-Marinas. Oficina Regional para América Latina y el Caribe del Programa de Naciones Unidas para el Medio Ambiente (PNUMA). Cartagena, Colombia, del 8 al 18 de noviembre de 2011. 